\title{
The universal separable metric space of Urysohn and isometric embeddings thereof in Banach spaces
}

\author{
by
}

\author{
M. Randall Holmes (Boise, Id.)
}

\begin{abstract}
This paper is an investigation of the universal separable metric space up to isometry $U$ discovered by Urysohn. A concrete construction of $U$ as a metric subspace of the space $C[0,1]$ of functions from $[0,1]$ to the reals with the supremum metric is given. An answer is given to a question of Sierpiński on isometric embeddings of $U$ in $C[0,1]$. It is shown that the closed linear span of an isometric copy of $U$ in a Banach space which contains the zero of the Banach space is determined up to linear isometry. The question of what Banach spaces can be embedded in a linear isometric fashion in this uniquely determined closed linear span of $U$ is investigated.
\end{abstract}

0. Introduction. A well-known result of elementary topology is the fact that certain topological spaces, such as the Hilbert cube, are "universal separable metric spaces" - such a space is a separable metric space which contains a homeomorph of each separable metric space. A less well-known result of metric topology is that there are universal separable metric spaces up to isometry; that is, there are separable metric spaces which contain an isometric copy of each separable metric space. The best-known theorem establishing the existence of a universal separable metric space up to isometry is the theorem of Banach and Mazur which asserts that $C[0,1]$, the space of continuous functions from $[0,1]$ to the reals with the supremum metric, is such a space (see [B], [B-P]; Banach and Mazur actually show that $C[0,1]$ is a universal separable Banach space up to linear isometry; we cite this result as Theorem 4 of Part III). But the first result of this kind was obtained by Urysohn (see [U]), who constructed a metric space $U$ of this kind which he proved can be characterized up to isometry by the fact that it is a universal separable metric space up to isometry which is complete and "metrically homogeneous with respect to finite sets". The definition of this property is given below.

Urysohn's construction of $U$ is highly abstract. We are able to present a more concrete construction of $U$ inside $C[0,1]$ (in the proof of Theorem 1 of 
Part II), using lemmas which relate isometric embeddings of $U$ in $C[0,1]$ to space-filling curves (Lemmas $0-4$ of Part II). In [H], Huhunaishvili refined some results of Urysohn, showing that certain properties which Urysohn proved of finite sets of points of $U$ can be established for compact subsets of $U$, making it possible to improve the characterization of $U$ (in $[\mathrm{J}$ ], Joiner improved a result on finite sets to Cauchy sequences; we give results of Urysohn, Joiner and Huhunaishvili in Part I). Here is our characterization of $U$.

THEOREM. $U$ is determined uniquely up to isometry by the fact that it is a separable metric space and any isometry from a subspace of a compact metric space $X$ into $U$ can be extended to the whole of $X$ (this is the Corollary to the Theorem of Part I).

Sierpiński, in [S], where he gave an "elementary" proof of the theorem of Banach and Mazur (a version is given as the proof of Theorem 1 of Part III below), pointed out that their result implies that $U$ can be embedded in $C[0,1]$. He asked whether there were simpler or more natural embeddings of $U$ in $C[0,1]$ than those given by the general procedure he used in his proof to embed an arbitrary separable metric space in $C[0,1]$, which made essential use of space-filling curves. We are able to answer this question essentially in the negative; in particular, any isometric embedding of $U$ in $C[0,1]$ is intimately related to a kind of space-filling curve.

TheOREM. For any isometric copy of $U$ in $C[0,1]$ containing $\mathbf{0}$, the constant zero function, we can define a map $F$ from $[0,1]$ to the space $U^{\prime}$ of Lipschitz functions on $U$ of norm $\leq 1$ which send $\mathbf{0}$ to 0 by $F(t)(f)=f(t)$, where $t \in[0,1]$ and $f$ is in the isometric copy of $U$. If we put the pointwise convergence topology on $U^{\prime}, F$ is a continuous path whose range includes $g$ or $-g$ for each $g \in U^{\prime}$. $U^{\prime}$ is a Hilbert cube (topologically), and F "half-fills" it (visits each point or its negative). The class of such paths $F$ corresponds exactly to the isometric embeddings of $U$ in $C[0,1]$ (this follows from the corollary to Lemma 5 of Part II, the Lemma of Part III, and Theorem 2 of Part III)

Finally, we can establish some surprising results about isometric embeddings of $U$ in Banach spaces.

THEOREM. All isometric copies of $U$ in Banach spaces which contain the zero element of the Banach space have the same closed linear span $\mathbf{U}$ up to linear isometry. Moreover, any finite collection of points of the copy of $U$ which does not include the zero is linearly independent (see Theorem 1 of Part IV).

We ask a natural question: is the separable Banach space $\mathbf{U}$ a universal separable Banach space up to linear isometry? This question turns out 
to be very hard to answer! We are able to embed the space $L_{1}$ of measurable functions on the reals with the integral norm in $\mathbf{U}$, and thus all two-dimensional Banach spaces (see Theorem 2 of Part IV and its Corollary). We can establish that $\mathbf{U}$ does not have a homogeneity property as a Banach space analogous to that of $U$ as a metric space (see Theorem 3 of Part IV). Whether $\mathbf{U}$ is universal for separable Banach spaces remains an open problem. $U$ is a "natural" object as a metric space; it is surprising that its uniquely determined linear closure as a Banach space seems to be much harder to work with.

Another question which the author thinks might be interesting but has not seriously attacked is whether $\mathbf{U}$ has a basis in the sense of the classical theory of Banach spaces. This question might be hard to answer, because $\mathbf{U}$ is defined in a way which has no analogue among the definitions of the Banach spaces usually studied.

A caution: in this paper, we are usually considering metric spaces and isometries or separable Banach spaces and linear isometries rather that topological spaces and homeomorphisms. If we mention "embeddings" without qualification, they should usually be taken to be isometric or linearly isometric. We adopt the convention that $d$ represents all metrics; we have no occasion in this paper to consider more that one metric on the same set.

Results are numbered independently in each part and referred to with the number of the part outside the part in which they reside.

\section{The crucial metric property and its consequences}

Definition. Let $X$ be a metric space, and let $A$ be a subset of $X$. A function $r$ from $A$ to the nonnegative reals will be called a possible combination of distances from $A$ if it satisfies the inequalities $|r(x)-r(y)| \leq$ $d(x, y) \leq r(x)+r(y)$ for each pair of points $x, y \in A$. We will refer to the set of possible combinations of distances from $A$ as $R(A)$. A possible combination of distances will be termed a possible combination of rational distances if all values in its range are rational; the set of possible combinations of rational distances from $A$ will be denoted by $R_{0}(A)$. We say that there are points at all possible combinations of (rational) distances from $A$ in $X$ when for each $r \in R(A)\left(R_{0}(A)\right)$ there is a point $p \in X$ such that $d(p, a)=r(a)$ for each $a \in A$.

A metric space $X$ is said to be (nearly) inflatable if for each finite subset $A$ of $X$ there are points at all possible combinations of (rational) distances from $A$. Note that an inflatable metric space must be nonempty-consider empty $A$.

ObSeRvation. A complete, inflatable, separable metric space $V$ must be a universal separable metric space up to isometry. 
Pr o of. Consider a countable metric space $\left\{a_{0}, a_{1}, a_{2}, \ldots\right\}$. There is certainly an isometry from $\left\{a_{0}\right\}$ into $V$. Given an isometry $I$ from $\left\{a_{0}, \ldots, a_{n}\right\}$ into $V$, construct an isometry $I^{\prime}$ from $\left\{a_{0}, \ldots, a_{n+1}\right\}$ into $V$ which extends $I$ as follows. The map $r$ which takes $I\left(a_{i}\right)$ to $d\left(a_{i}, a_{n+1}\right)$ for $0 \leq i \leq n$ is a possible combination of distances from $\left\{a_{0}, \ldots, a_{n}\right\}$; by inflatability of $V$, we can choose $I^{\prime}\left(a_{n+1}\right)$ so that $d\left(I\left(a_{i}\right), I^{\prime}\left(a_{n+1}\right)\right)=r\left(I\left(a_{i}\right)\right)=d\left(a_{i}, a_{n+1}\right)$ for $0 \leq i \leq n$. By induction, initial segments of a countable metric space can be uniformly isometrically embedded in $V$, so any countable metric space can be embedded in $V$. Thus, any separable metric space can be isometrically embedded in $V$ : let $X$ be a separable metric space; a countable dense subset $D$ of $X$ can be isometrically embedded in $V$, because $V$ is inflatable; thus, the completion of $D$, which is isometric to the completion of $X$, can be isometrically embedded in $V$, because $V$ is complete. Since $V$ is separable and contains an isometric copy of each separable metric space, $V$ is a universal separable metric space up to isometry.

We will show below (in Part II) that there is a complete, inflatable separable metric space $U$. Urysohn proved in $[\mathrm{U}]$ that there is exactly one such metric space, up to isometry.

Lemma. Let $A$ be a finite subset of a metric space $X$. For each real number $e>0$ and strictly positive function $r \in R(A)$, there is a function $r^{\prime} \in R_{0}(A)$ such that $\left|r(a)-r^{\prime}(a)\right|<$ e for each $a \in A$.

Proof. Let $m$ be the number of distinct values in the range of $r$. Let $e^{\prime}$ be $\min \{e, d\}$, where $d$ is the smallest nonzero absolute difference between values in the range of $r$. Replace the largest value in the range of $r$, wherever it occurs, with a rational value larger by less than $e^{\prime} / m$. Replace the secondlargest value in the range of $r$ by a rational value larger by an amount strictly between $e^{\prime} / m$ and $2 e^{\prime} / m$. In general, replace the $i$ th largest value in the range of $r$ by a rational value larger by an amount strictly between $(i-1) e^{\prime} / m$ and $i e^{\prime} / m$. Observe that the substitution for values in the range of $r$ is monotone, because, while smaller values are increased by a larger amount, no value is increased sufficiently to exceed the original value of the next larger value. The resulting function is the desired $r^{\prime}$. It is clearly rational-valued and differs by less than $e$ from $r$; we need to verify that it is a possible combination of distances from $A$. Indeed, our procedure increased sums of possible distances and decreased positive absolute differences of possible distances (leaving zero differences unchanged): $\left|r^{\prime}(x)-r^{\prime}(y)\right| \leq$ $|r(x)-r(y)| \leq d(x, y) \leq r(x)+r(y) \leq r^{\prime}(x)+r^{\prime}(y)$.

Proposition. The following are equivalent for a complete metric space $X$ :

(a) X contains a dense nearly inflatable subset. 
(b) $X$ is inflatable.

(c) For each compact subspace $A$ of $X$, there are points at all possible combinations of distances from $A$ in $X$.

Proof. That (c) implies (b) and (b) implies (a) are obvious. It suffices to show that (a) implies (c).

Suppose that $X$ is a metric space with a nearly inflatable dense subset $D$. We will construct a sequence $\left\{p_{1}, p_{2}, \ldots\right\}$ of points of $X$, a sequence $\left\{A_{1}, A_{2}, \ldots\right\}$ of compact subsets of $X$, a sequence $\left\{r_{1}, r_{2}, \ldots\right\}$ with $r_{i}$ an element of $R\left(A_{i}\right)$, and a sequence $\left\{B_{1}, B_{2}, \ldots\right\}$ of finite subsets of $D$.

Let $e$ be a positive real number and set $A_{1}=A$. Let $r=r_{1}$ be a strictly positive element of $R(A)$. Extend $r$ to the whole of $X$ by defining $r(x)=\max _{a \in A}|r(a)-d(a, x)|$ for each $x \in X$; it is easy to verify that this is a strictly positive element of $R(X)$. Let $B_{1}$ be a finite subset of $D$ which contains elements within $e / 6$ of each element of $A$. Use the Lemma to find an $r^{\prime} \in R_{0}\left(B_{1}\right)$ which differs from $r \mid B_{1}$ by less than $e / 6$; there is a point $p_{1} \in D$ such that $d\left(p_{1}, b\right)=r^{\prime}(b)$ for each $b \in B_{1}$. Observe that $d\left(p_{1}, a\right)$, for $a \in A$, differs from $d\left(p_{1}, b\right)=r^{\prime}(b)$ for some $b \in B_{1}$ by less than $e / 6$, $\left|r^{\prime}(b)-r(b)\right|<e / 6$, and $|r(b)-r(a)|<e / 6$, so $\left|d\left(p_{1}, a\right)-r(a)\right|<e / 2$.

We continue the construction inductively as follows. When we have constructed $A_{1}$ and $p_{i}$, where for each $a \in A_{i},\left|d\left(a, p_{i}\right)-r_{i}(a)\right|<e / 2^{i}$, we define $A_{i+1}$ as $A_{i} \cup\left\{p_{i}\right\}$ and $r_{i+1}$ as $r_{i} \cup\left\{\left(p_{i}, e / 2^{i}\right)\right\}$, which is easily seen to belong to $R\left(A_{i+1}\right)$. We extend $r_{i+1}$ to $X$ as above. We choose $B_{i+1}$ to be a finite subset of $D$ containing points within $1 /\left(3 \cdot 2^{i+1}\right)$ of each point of $A_{i+1}$. We choose $r^{\prime} \in R_{0}\left(B_{i+1}\right)$ approximating $r_{i+1}$ within $1 /\left(3 \cdot 2^{i+1}\right)$ and let $p_{i+1}$ be a point in $D$ at distance $r^{\prime}(b)$ from each point $b$ of $B_{i+1}$. We show as above that $\left|d\left(p_{i+1}, a\right)-r_{i+1}(a)\right|<1 / 2^{i+1}$ for each $a \in A_{i+1}$. This allows us to continue the induction. Note that $d\left(p_{i}, p_{i+1}\right)$ differs by less than $1 / 2^{i+1}$ from $r_{i+1}\left(p_{i}\right)=1 / 2^{i}$.

The sequence of $p_{i}$ 's is a Cauchy sequence, so must converge to a point $p$, and it is obvious that $d(a, p)=r(a)$ for each $a \in A$, since the sequence of $d\left(a, p_{i}\right)$ 's must converge to $d(a, p)$, and $\left|d\left(a, p_{i}\right)-r(a)\right|<1 / 2^{i}$.

THEOREM (following Urysohn as extended by Huhunaishvili). The following are equivalent for a complete separable metric space $U$ :

(a) $U$ is inflatable.

(b) If $X$ is a separable metric space and $A$ its compact subset, then any isometric embedding of $A$ into $U$ can be extended to an isometric embedding of $X$ into $U$.

(c) If $V$ is a complete inflatable separable metric space and $A$ is a compact subset of $V$, then any isometric embedding of $A$ into $U$ can be extended to an isometric embedding of $V$ onto $U$. 
COROLlary. There is at most one complete inflatable separable metric space $U$, up to isometry, and it is characterized up to isometry by the facts that it is a separable metric space and that any isometry from a subset of a compact metric space $X$ into $U$ can be extended to an isometry from the whole of $X$ into $U$.

Proof of Theorem and Corollary. We prove first that (a) implies (c).

Let $U$ and $V$ be complete inflatable separable metric spaces. Let the compact subset $A$ of $V$ be the domain of any isometry $I$ into $U$. Let $\left\{a_{1}, a_{2}, \ldots\right\}$ be a countable dense subset of $V-A$. Let $\left\{b_{1}, b_{2}, \ldots\right\}$ be a countable dense subset of $U-I[A]$. Choose a point $p \in U$ such that $d(p, I(x))=d\left(a_{1}, x\right)$ for each $x \in A$; such a point exists by part (c) of the Proposition. Choose a point $q \in V$ such that $d(q, x)=d\left(b_{1}, I(x)\right)$ for each $x \in A$. Extend $I$ by setting $I\left(a_{1}\right)=p$ and $I(q)=b_{1}$. This completes the first step of the construction. At the $i$ th step, when $I\left(a_{j}\right)$ and $I^{-1}\left(b_{j}\right)$ have been defined for each $j<i$, choose $p$ such that $d(p, I(x))=d\left(a_{i}, x\right)$ for each $x$ in the domain of $I$ (including points of $A, a_{j}$ 's for $j<i$, and $I^{-1}\left(b_{j}\right)$ 's for $j<i$ ). Choose $q$ such that $d(q, x)=d\left(b_{i}, I(x)\right)$ for each $x$ in the same domain. Extend $I$ by setting $I\left(a_{i}\right)=p$ and $I(q)=b_{i}$. This process enables us to extend $I$ to an isometry from a dense subset of $V$ onto a dense subset of $U$, and thus to an isometry from $V$ onto $U$.

We can now prove that (a) implies (b). Suppose that $A$, a compact subset of a separable metric space $X$, can be embedded isometrically in $U$. We know (by the Observation above) that $X$ can be embedded isometrically in any complete inflatable separable $V$. Thus, we can consider the space $X$ to be included in $V$, and use the proof that (a) implies (c) to show that the embedding of $A$ into $U$ can be extended to an embedding of $V$ onto $U$, which can be restricted to an embedding of $X$ into $U$ extending the original embedding of $A$.

That (b) implies (a) is immediate. That (c) implies (a) and the Corollary follow easily once the existence of a complete inflatable metric space is established (see below). The alternate characterization of $U$ holds by the Theorem. To see that the property given implies completeness, consider an isometric embedding of a Cauchy sequence in $U$; it could be extended to an isometric embedding of the Cauchy sequence with its limit point. To see that the property given implies inflatability, consider an embedding of all but one point of a finite metric space into $U$.

The results listed above were established by Urysohn and Huhunaishvili. Urysohn used the property which we call "inflatability" to motivate his construction of $U$. He also used it to prove that $U$ is "metrically homogeneous with respect to finite sets" - that every isometry from a finite subset of $U$ 
to a finite subset of $U$ extends to an isometry from $U$ onto $U$. Urysohn proved that $U$ is uniquely characterized up to isometry by the facts that it is complete, metrically homogeneous with respect to finite sets, and is a universal separable metric space up to isometry; implicit in his proof is an argument that $U$ is uniquely characterized as complete, inflatable and separable. Joiner proved that $U$ is metrically homogeneous with respect to Cauchy sequences, but Huhunaishvili had already shown that $U$ is metrically homogeneous with respect to compact sets (part (c) with $U=V$ ), from which the extensions of properties of $U$ from finite to compact sets easily follow. The fact that Joiner presents in $[\mathrm{J}]$ an easy consequence of the work presented almost twenty years earlier in $[\mathrm{H}]$ was an indication to us that it was advisable to summarize all this work here.

An interesting question raised by Urysohn is whether completeness can be dropped from his characterization of $U$-must any universal separable metric space up to isometry which is metrically homogeneous with respect to finite sets be isometric to $U$ ? This question has been answered in the negative in the unpublished note [Z] of Martin Ziegler. It seems that E. Rothacker independently found the results of Huhunaishvili in 1976, but did not publish his findings.

II. The construction of $U$ inside $C[0,1]$. In this part we will construct an inflatable subspace of $C[0,1]$, the space of continuous functions from $[0,1]$ to the reals with the supremum metric $d(f, g)=\sup _{t \in[0,1]} \mid f(t)-$ $g(t) \mid . C[0,1]$ is known to be a universal separable metric space up to isometry by a theorem of Banach and Mazur (see $[\mathrm{B}],[\mathrm{B}-\mathrm{P}]$ and Part III below). The construction provides another proof of this result.

Definition. If $S$ is a set of functions in $C[0,1]$, and $r$ is a possible combination of distances from $S$, we define the functions $A_{r}$ and $B_{r}$ in $C[0,1]$ by $A_{r}(x)=\sup _{f \in S} f(x)-r(f)$ and $B_{r}(x)=\inf _{f \in S} f(x)+r(f)$. Note that $A_{r}(x)$ will be the smallest value that a function with distance $r(f)$ from each $f \in S$ could have at $x$, and $B_{r}(x)$ will be the largest. $A_{r}$ and $B_{r}$ can only be assumed to be continuous functions if $S$ is finite or satisfies other special conditions. The notation is adequate because $S$ can be determined from $r$.

LEMMA 0. If $r$ is a possible combination of distances from $\left\{f_{1}, \ldots, f_{n}\right\}$, a finite set of functions in $C[0,1]$, then $A_{r}$ and $B_{r}$ are continuous functions, $A_{r}(x) \leq B_{r}(x)$ for each $x \in[0,1], d\left(A_{r}, f_{i}\right) \leq r\left(f_{i}\right)$ for each $i \leq n$, and $d\left(B_{r}, f_{i}\right) \leq r\left(f_{i}\right)$ for each $i \leq n$.

Proof. Omitted.

LEMMA 1. There are points at all possible combinations of distances from a finite subset $\left\{f_{1}, \ldots, f_{n}\right\}$ of $C[0,1]$ exactly if for each $i \leq n$ there is an 
$s_{i}= \pm 1$ and an $x_{i} \in[0,1]$ such that $f_{j}\left(x_{i}\right)=f_{i}\left(x_{i}\right)+s_{i} d\left(f_{i}, f_{j}\right)$ for each $j \leq n$, in other words, exactly if for each $f_{i}$ there is $x_{i} \in[0,1]$ such that each other $f_{j}$ takes on a value at $x_{i}$ as large as its distance from $f_{i}$ permits, or such that each other $f_{j}$ takes on a value at $x_{i}$ as small as its distance from $f_{i}$ permits.

Proof. Let $D$ be the diameter of $\left\{f_{1}, \ldots, f_{n}\right\}$. Let $r_{i}$ be the function which takes each $f_{j}$ to $(3 / 2) D-d\left(f_{i}, f_{j}\right)$. It is straightforward to establish that $r_{i}$ is a possible combination of distances from $\left\{f_{1}, \ldots, f_{n}\right\}$. Thus, if there is a point at every possible combination of distances from the $f_{i}$ 's, there is a function $g_{i} \in C[0,1]$ such that $d\left(g_{i}, f_{j}\right)=(3 / 2) D-d\left(f_{i}, f_{j}\right)$ for each $j$. In particular, $d\left(g_{i}, f_{i}\right)=(3 / 2) D$. Thus, there is a point $x_{i}$ such that $\left|g_{i}\left(x_{i}\right)-f_{i}\left(x_{i}\right)\right|=(3 / 2) D$, i.e., $g_{i}\left(x_{i}\right)-f_{i}\left(x_{i}\right)=s_{i}(3 / 2) D$, where $s_{i}= \pm 1$. Suppose $s_{i}=1$. Then $g_{i}\left(x_{i}\right)=f_{i}\left(x_{i}\right)+(3 / 2) D$, and so $f_{i}\left(x_{i}\right)+d\left(f_{i}, f_{j}\right) \geq$ $f_{j}\left(x_{i}\right) \geq g_{i}\left(x_{i}\right)-d\left(g_{i}, f_{j}\right)=f_{i}\left(x_{i}\right)+(3 / 2) D-\left((3 / 2) D-d\left(f_{i}, f_{j}\right)\right)=f_{i}\left(x_{i}\right)+$ $d\left(f_{i}, f_{j}\right)$, for each $j \leq n$. The proof for $s_{i}=-1$ is exactly analogous, with suitable interchange of signs and senses of inequalities.

If for each $f_{i}$ there are $x_{i}$ and $s_{i}$ as described, and $r$ is a possible combination of distances from the $f_{i}$ 's, choose a continuous function $h$ from $[0,1]$ to $[-1,1]$ such that $h\left(x_{i}\right)=s_{i}$ for each $i \leq n$. Define $g(x)=$ $((1-h(x)) / 2) A_{r}(x)+((1+h(x)) / 2) B_{r}(x)$. Since $A_{r}(x) \leq g(x) \leq B_{r}(x)$ for all $x$ by Lemma 0 and the fact that $g$ is a convex combination of $A_{r}$ and $B_{r}, d\left(g, f_{i}\right) \leq r\left(f_{i}\right)$ for each $i \leq n$. Now consider $g\left(x_{i}\right)$. We have $h\left(x_{i}\right)=s_{i}$, so if $s_{i}=1, g\left(x_{i}\right)=B_{r}\left(x_{i}\right)$, and if $s_{i}=-1, g\left(x_{i}\right)=A_{r}\left(x_{i}\right)$. If $s_{i}=1$, then $B_{r}\left(x_{i}\right)$ is the minimum of expressions of the form $f_{j}\left(x_{i}\right)+r\left(f_{j}\right)=$ $f_{i}\left(x_{i}\right)+d\left(f_{i}, f_{j}\right)+r\left(f_{j}\right) \geq f_{i}\left(x_{i}\right)+d\left(f_{i}, f_{i}\right)+r\left(f_{i}\right)=f_{i}\left(x_{i}\right)+r\left(f_{i}\right)$, because $r\left(f_{i}\right)-r\left(f_{j}\right) \leq d\left(f_{i}, f_{j}\right)$ by the definition of a possible combination of distances. Thus $g\left(x_{i}\right)=f_{i}\left(x_{i}\right)+r\left(f_{i}\right)$. The proof that $g\left(x_{i}\right)=A_{r}\left(x_{i}\right)=$ $f_{i}\left(x_{i}\right)-r\left(f_{i}\right)$ when $s_{i}=-1$ is analogous. It follows that $d\left(g, f_{i}\right)=r\left(f_{i}\right)$ for each $i \leq n$.

It is an immediate consequence of this lemma that $C[0,1]$ is not inflatable. If it were, then there would be points at every possible combination of distances from every finite subset of $C[0,1]$. Consider the constant functions $f_{1}(x)=0, f_{2}(x)=1$, and $f_{3}(x)=2$. If there were points at every possible combination of distances from these, there would be a point $x_{2} \in[0,1]$ such that $f_{1}\left(x_{2}\right)$ and $f_{3}\left(x_{2}\right)$ would either both be greater than $f_{2}\left(x_{2}\right)$ or both be less than $f_{2}\left(x_{2}\right)$, by the Lemma. This is absurd. A possible combination of distances which is not realized is distance 1 from $f_{1}$, distance 1 from $f_{2}$, and distance 1 from $f_{3}$ (since the only function at distance 1 from $f_{1}$ and $f_{3}$ is $f_{2}$ itself).

The condition of Lemma 1 is a necessary but not a sufficient condition for a finite collection of functions in $C[0,1]$ to be a subset of an inflatable 
metric subspace of $C[0,1]$. A stronger necessary condition, which is still not quite sufficient, will be given now. It will be assumed from this point on that the constant zero function $\mathbf{0}$ belongs to any inflatable subset of $C[0,1]$ discussed; all other inflatable sets can be obtained from inflatable sets containing $\mathbf{0}$ by a simple translation. For each $f \in C[0,1]$, we define $|f|=d(\mathbf{0}, f)$. (We may write this $\|f\|$ when we want to be careful to avoid confusion between norms of functions and absolute values of numbers.)

Definition. A possible combination of values for a set $A$ of functions in $C[0,1]$ is a function $r$ from $A$ to the reals such that $|r(f)| \leq|f|$ for each $f \in A$ and $|r(f)-r(g)| \leq d(f, g)$ for each $f, g \in A$. This is equivalent to the notion of "restriction to $A$ of a Lipschitz function with constant $\leq 1$ on $A \cup\{\mathbf{0}\}$ which sends $\mathbf{0}$ to 0 ". Note that for any $x \in[0,1]$, the function $r_{x}$ which takes each $f \in A$ to $f(x)$ is a possible combination of values for $A$. A set $A$ of functions in $C[0,1]$ takes on half of all possible combinations of values if and only if for each possible combination of values $r$ for $A$ there is an $x \in C[0,1]$ and an $s= \pm 1$ such that $f(x)=\operatorname{sr}(f)$ for each $f \in A$.

LEMMA 2. If a finite subset $\left\{f_{1}, \ldots, f_{n}\right\}$ of $C[0,1]$ is a subset of an inflatable metric subspace of $C[0,1]$ which contains $\mathbf{0}$, then it takes on half of all possible combinations of values.

Proof. Let $r$ be a possible combination of values for the $f_{i}$ 's. Let $D$ be the diameter of the set consisting of the $f_{i}$ 's and $\mathbf{0}$. Then the function which sends 0 to $(3 / 2) D$ and each $f_{i}$ to $(3 / 2) D+r\left(f_{i}\right)$ is a possible combination of distances from this set. Since $\mathbf{0}$ and the $f_{i}$ 's all belong to the same inflatable subset of $C[0,1]$, there is a function $g$ in the inflatable subset of $C[0,1]$ such that $d(g, \mathbf{0})=(3 / 2) D$ and $d\left(g, f_{i}\right)=(3 / 2) D+r\left(f_{i}\right)$ for each $i \leq n$. There are points at all possible combinations of distances from the set consisting of $\mathbf{0}, g$ and the $f_{i}$ 's, since it is a finite subset of an inflatable space. By Lemma 1 , there is an $x_{g} \in[0,1]$ and an $s_{g}= \pm 1$ such that $\mathbf{0}\left(x_{g}\right)=g\left(x_{g}\right)+s_{g}(3 / 2) D$ and $f_{i}\left(x_{g}\right)=g\left(x_{g}\right)+s_{g}\left((3 / 2) D+r\left(f_{i}\right)\right)$. Since $\mathbf{0}$ is the constant zero function, we have $g\left(x_{g}\right)=-s_{g}(3 / 2) D$, whereupon we have $f_{i}\left(x_{g}\right)=s_{g} r\left(f_{i}\right)$.

The converse of Lemma 2 is not true; not all sets of functions which take on half of all possible combinations of values are subsets of inflatable sets in $C[0,1]$ - but we are on the right track.

Definition. If $S$ is a metric subspace of $C[0,1]$ and $g$ is a function in $C[0,1]$ such that $|g|=1, d(f, g)=1+|f|$ for each $f \in S$, and $S \cup\{g\}$ takes on half of all possible values, we say that $g$ inflates $S$.

Remark. A function $g$ inflates the subset $A$ of $C[0,1]$ iff $|g|=1$ and for any $t \in[-1,1]$ and for any $r \in R(A)$ there exist $s= \pm 1$ and $x \in[0,1]$ such that $g(x)=$ st and $f(x)=\operatorname{sr}(f)$ for each $f \in A$. 
Lemma 3. Suppose $g$ inflates $S$ and $h$ is a map from $[-1,1]$ onto $[-1,1]^{2}$, with $h(t)=\left(h_{1}(t), h_{2}(t)\right)$ and $h$ odd, that is, $h(-t)=\left(-h_{1}(t),-h_{2}(t)\right)$. Define the functions $g^{\prime}$ and $g^{\prime \prime}$ by $g^{\prime}(t)=h_{1}(g(t))$ and $g^{\prime \prime}(t)=h_{2}(g(t))$. If $T$ is any set of functions such that $T$ takes on half of all possible combinations of values and the value of any function in $T$ is an odd function of the values of $g^{\prime \prime}$ and the functions of $S$, then $g^{\prime}$ inflates $T$. In particular, $g^{\prime}$ inflates $S$.

Proof. Certainly $\left|g^{\prime}\right|=1$ and, given $r \in R(T)$ and $t \in[-1,1]$, there exist $s= \pm 1$ and $x \in[0,1]$ such that $g(x)=s t$ and $f(x)=\operatorname{sr}(f)$ for $f \in T$. Take $u \in[0,1]$ so that $h(u)=\left(s t, g^{\prime \prime}(x)\right)$ and apply the Remark to get $y \in[0,1]$ and $s^{\prime}= \pm 1$ such that $f(y)=s^{\prime} f(x)=s^{\prime} s r(f)$ for $f \in S$ and $g(y)=s^{\prime} u$. Then $g^{\prime}(y)=h_{1}(g(y))=h_{1}\left(u s^{\prime}\right)=s^{\prime} s t$. Similarly, the values of the functions in $T$ being odd functions of the values of $g^{\prime \prime}$ and of the values of the functions in $S$, it follows easily that $f(y)=f(x)=s^{\prime} s r(f)$ for $f \in T$. By the Remark, it follows that $g^{\prime}$ inflates $T$.

Lemma 4. Given a metric subspace $S$ of $C[0,1]$, a function $g$ which inflates $S$, and a possible combination of distances $r$ from a finite subset $\left\{f_{1}, \ldots, f_{n}\right\}$ of $S$, we can find a function $f_{n+1} \in C[0,1]$ such that $d\left(f_{i}, f_{n+1}\right)=r\left(f_{i}\right)$ for each $i \leq n$, and a function $g^{\prime}$ which inflates $S \cup$ $\left\{f_{n+1}\right\}$.

Proof. We extend $r$ to the whole of $S$ by the definition $r(f)=$ $\min _{i \leq n} d\left(f, f_{i}\right)+r\left(f_{i}\right)$. It is straightforward to check that the functions $A_{r}$ and $B_{r}$ are unaffected by this extension.

Let $h=\left(h_{1}, h_{2}\right)$ be an odd map from $[-1,1]$ onto $[-1,1]^{2}$. Define $g^{\prime}(x)=h_{1}(g(x)), g^{\prime \prime}(x)=h_{2}(g(x))$, and $f_{n+1}(x)=\left(\left(1-g^{\prime \prime}(x)\right) / 2\right) A_{r}(x)+$ $\left(\left(1+g^{\prime \prime}(x)\right) / 2\right) B_{r}(x)$. Since $A_{r}(x) \leq f_{n+1}(x) \leq B_{r}(x)$ for each $x$, we have $d\left(f_{i}, f_{n+1}\right) \leq r\left(f_{i}\right)$ for each $i \leq n$.

Fix $i \leq n$. The function which takes $f_{j}$ to $d\left(f_{i}, f_{j}\right)-\left|f_{i}\right|$ and $g$ to any value $t \in[-1,1]$ is a possible combination of values for these functions. Choose $t$ so that $h_{2}(t)=1$. Thus there is an $x_{i} \in[0,1]$ and an $s_{i}= \pm 1$ such that $f_{j}\left(x_{i}\right)=s_{i}\left(d\left(f_{i}, f_{j}\right)-\left|f_{i}\right|\right)$ and $g\left(x_{i}\right)=s_{i} t$, which implies that $g^{\prime \prime}\left(x_{i}\right)=h_{2}\left(s_{i} t\right)=s_{i}$. It follows that if $s_{i}=1, f_{n+1}\left(x_{i}\right)=B_{r}\left(x_{i}\right)$, and if $s_{i}=-1, f_{n+1}\left(x_{i}\right)=A_{r}\left(x_{i}\right)$. In the case where $s_{i}=1$, we have $f_{n+1}\left(x_{i}\right)=$ $B_{r}\left(x_{i}\right)$, which is the minimum of expressions of the form $f_{j}\left(x_{i}\right)+r\left(f_{j}\right)=$ $d\left(f_{i}, f_{j}\right)-\left|f_{i}\right|+r\left(f_{j}\right) \geq d\left(f_{i}, f_{i}\right)-\left|f_{i}\right|+r\left(f_{i}\right)=f_{i}\left(x_{i}\right)+r\left(f_{i}\right)$, because $d\left(f_{i}, f_{j}\right) \geq r\left(f_{i}\right)-r\left(f_{j}\right)$. This forces $d\left(f_{i}, f_{n+1}\right)=r\left(f_{i}\right)$. The proof for the case $s_{i}=-1$ is symmetrical.

Now we need to show that $S \cup\left\{f_{n+1}\right\}$ takes on half of all possible combinations of values. Let $R$ be such a possible combination of values. Let $A_{R}=\max _{f \in S} R(f)-r(f)$ and let $B_{R}=\min _{f \in S} R(f)+r(f)$. Then $A_{R}$ and $B_{R}$ are lower and upper bounds respectively on the possible values of $R\left(f_{n+1}\right)$ given the values of $R(f)$ for $f \in S$. Let $c=$ 
$\left(R\left(f_{n+1}\right)-A_{R}\right) /\left(B_{R}-A_{R}\right)$. Let $C=2 c-1 . \quad C$ is a quantity between -1 and 1 whose value is determined in an affine manner by the position of $R\left(f_{n+1}\right)$ in the interval $\left[A_{R}, B_{R}\right]$. Now define $R^{\prime}$, a possible combination of values for $\left\{f_{1}, \ldots, f_{n}, g\right\}$, by $R^{\prime}\left(f_{i}\right)=R\left(f_{i}\right)$ for each $i \leq n$ and $R^{\prime}(g)=t$, where $h_{2}(t)=C$. Thus, there is an $x \in[0,1]$ and an $s= \pm 1$ such that $f_{i}(x)=s R\left(f_{i}\right)$ for each $i \leq n$, and $g(x)=t$, so that $g^{\prime \prime}(x)=s C$. When $s=1, A_{r}(x)=A_{R}$ and $B_{r}(x)=B_{R}$, and, symmetrically, $A_{r}(x)=-B_{R}$ and $B_{r}(x)=-A_{R}$ when $s=-1$. In the case where $s=1$, we get $f_{n+1}(x)=((1-C) / 2) A_{R}+((1+C) / 2) B_{R}=R\left(f_{n+1}\right)$ by choice of $C$. When $s=-1$, we get $f_{n+1}(x)=-R\left(f_{n+1}\right)$ in a symmetrical fashion. Thus $S \cup\left\{f_{n+1}\right\}$ takes on half of all possible combinations of values. Since the value of $f_{n+1}$ is an odd function of the values of $S$ and $g^{\prime \prime}, g^{\prime}$ inflates $S \cup\left\{f_{n+1}\right\}$ by Lemma 3 .

LEMma 5. If $A$ is a subset of $C[0,1]$, and any finite subset of $A$ takes on half of all possible combinations of values, then $A$ takes on half of all possible combinations of values.

Proof. Let $A_{0}$ be a countable dense subset of $A$. Let $A_{n}$, for each positive integer $n$, be a set with $n$ elements, with $A_{n} \subset A_{n+1}$ for each $n$ and $\bigcup_{n>0} A_{n}=A_{0}$. Let $f$ be a possible combination of values for $A$. For each $n$, there is a $t_{n} \in[0,1]$ and an $s_{n}= \pm 1$ such that $a\left(t_{n}\right)=s_{n} f(a)$ for each $a \in A_{n}$. Let $s= \pm 1$ be chosen to equal $s_{n}$ for infinitely many $n$; let $T$ be the set of $t_{n}$ 's for which $s_{n}=s$. Let $t$ be a cluster point of $T$; it is obvious that $a(t)=s f(t)$ for each $a \in A$.

Corollary to Lemma 5. An inflatable metric subspace of $C[0,1]$ which contains $\mathbf{0}$ takes on half of all possible combinations of values.

Note that Lemmas $0-4$ are all true if we use instead of $C[0,1]$ any space of functions from a set to the reals with the supremum metric in which the distance $d(f, g)$ between any two functions in the space is actually equal to $|f(x)-g(x)|$ for some $x$ in the set. In particular, $C[0,1]$ can be replaced by the space of continuous functions from any compact metric space to the reals under the supremum metric (Lemma 5 will also hold in this case).

Now we will prove that there is a complete inflatable metric subspace of $C[0,1]$, which will be a universal separable metric space up to isometry. This also implies that $C[0,1]$ itself is a universal separable metric space up to isometry.

TheOREM 1. There is a complete inflatable metric subspace $U$ of $C[0,1]$.

P r o of. Let $X$ be any finite or countable metric subspace of $C[0,1]$ such that there is a function $g$ which inflates $X$. There are such subspaces: for instance, let $h=\left(h_{1}, h_{2}\right)$ be a map from $[0,1]$ onto $[-1,1]^{2}$, let $X=\left\{h_{1}\right\}$ and let $g=h_{2}$. 
We construct a countable metric subspace $X_{*}$ of $C[0,1]$ such that there are points in $X_{*}$ at every possible combination of rational distances from each finite subset of $X$, and there is a function $g^{\prime}$ which inflates $X_{*}$.

Let $h=\left(h_{1}, h_{2}\right)$ be an odd map from $[-1,1]$ onto $[-1,1]^{2}$. Define $g^{\prime}$ and $g_{1}$ by $g^{\prime}(x)=h_{1}(g(x))$ and $g_{1}(x)=h_{2}(g(x))$ for each $x \in[0,1]$. List all possible combinations of rational distances (possible combinations of distances whose ranges lie entirely in the positive rationals) from finite subsets of $X$; these make up a countable set $\left\{r_{1}, r_{2}, \ldots\right\}$. The function $g_{1}$ inflates $X$ by Lemma 3 ; thus, by Lemma 4 , there is a function $f_{1} \in C[0,1]$ such that $d\left(f, f_{1}\right)=r_{1}(f)$ for each $f$ in the domain of $r_{1}$, and a function $g_{2}$ which inflates $X \cup\left\{f_{1}\right\}$. By the second part of Lemma $3, g^{\prime}$ also inflates $X \cup\left\{f_{1}\right\}$, if $f_{1}$ and $g_{2}$ are constructed as described in Lemma 4. Repeat this process, at the $i$ th step finding a function $f_{i}$ such that $d\left(f, f_{i}\right)=r_{i}(f)$ for each $f$ in the domain of $r_{i}$ and a function $g_{i+1}$ which inflates $X \cup\left\{f_{1}, \ldots, f_{n}\right\}$, with $g^{\prime}$ also inflating $X \cup\left\{f_{1}, \ldots, f_{n}\right\}$, since the values of each $f_{i}$ are odd functions of the values of the functions in $X$ and the value of $g^{\prime \prime}$, if the $f_{i}$ 's and $g_{i}$ 's are constructed as in Lemma 4.

$X \cup\left\{f_{1}, f_{2}, \ldots\right\}$ is the desired $X_{*}$. By Lemma 5 and the construction, $g^{\prime}$ inflates $X_{*}$. It is obvious from the construction that $X_{*}$ contains points at all possible combinations of rational distances from each finite subset of $X$, and that $X_{*}$ is countable.

We construct a space $X_{i}$ for each positive integer $i$, with $X_{1}=X$, $X_{2}=X \cup X_{*}$, and $X_{i+1}=X_{i} \cup\left(X_{i}\right)_{*}$ for each positive integer $i$. The union $\bigcup_{i \in \mathbb{N}} X_{i}$ will be denoted by $U_{0}$. It is clear that $U_{0}$ contains points at every possible combination of rational distances from each finite subset of $U_{0}$; any finite subset of $U_{0}$ is a subset of some $X_{i}$, and $X_{i+1}$ contains points at every possible combination of rational distances from each finite subset of $X_{i}$. Let $U$ be the completion of $U_{0} ; U$ is a complete inflatable separable metric space by the Proposition of Part I.

THEOREM 2. A finite set of functions $\left\{f_{1}, \ldots, f_{n}\right\}$ is a subset of an inflatable subset of $C[0,1]$ containing $\mathbf{0}$ if and only if there is a function $g$ which inflates $\left\{f_{1}, \ldots, f_{n}\right\}$.

Proof. If $\left\{f_{1}, \ldots, f_{n}\right\}$ is a subset of an inflatable subset $V$ of $C[0,1]$, then there are points in $V$ at all possible combinations of distances from $\left\{\mathbf{0}, f_{1}, \ldots, f_{n}\right\}$. The function $r$ which takes $\mathbf{0}$ to 1 and $f_{i}$ to $1+\left|f_{i}\right|$ for each $i \leq n$ is a possible combination of distances. Thus, there is a function $g \in V$ at distance 1 from $\mathbf{0}$ and distance $1+\left|f_{i}\right|$ from $f_{i}$ for each $i$. Since $\left\{f_{1}, \ldots, f_{n}, g\right\}$ is a subset of the inflatable subset $V$ of $C[0,1]$, it takes on half of all possible combinations of values.

Suppose $\left\{f_{1}, \ldots, f_{n}, g\right\}$ takes on half of all possible combinations of values, $|g|=1$, and $d\left(g, f_{i}\right)=1+\left|f_{i}\right|$ for each $i \leq n$. We can suppose without 
loss of generality that $f_{1}=\mathbf{0}$, because if any finite subset of $C[0,1]$ has these properties, its union with $\{\mathbf{0}\}$ has them as well. Carry out the construction of $U$ in Theorem 1, using the set of $f_{i}$ 's as $X$ and $g$ as $g$, to construct a complete inflatable metric subspace of $C[0,1]$ containing the finite set.

A corollary of Theorem 2 is that a function is an element of an inflatable subset of $C[0,1]$ if and only if it is a component of a map from $[0,1]$ onto a square $[-a, a]^{2}$ whose range includes $(x, y)$ or $(-x,-y)$ for each $x, y \in$ $[-a, a]$. We call such a map a half-square-filler. The corollary is proved by examination of the case of Theorem 2 where the set of functions has only one element; this implies immediately that the map $\left(f_{1}, g\right)$ "half-fills" the product of $\left[-\left|f_{1}\right|,\left|f_{1}\right|\right]$ and $[-1,1]$; replace $g$ by $\left|f_{1}\right| g$ to get a halfsquare-filler with first component $f_{1}$. The converse is established by first linearly compressing a half-square-filler to a map "half-filling" a product one of whose terms is $[-1,1]$, then observing that the components of such a map satisfy the conditions of the Theorem.

We remark without the (tedious) proof that a slight modification of the construction proves "reversible"; every copy of $U$ in $C[0,1]$ which contains $\mathbf{0}$ is obtainable by the modified construction, by choosing parameters of the construction appropriately. The modification is to use as possible combinations of rational distances in the construction of each $X_{*}$ only those in which the inequalities are strict. It then proves possible to recover the functions used in the construction from an isometric copy of the modified $U_{0}$ in $C[0,1]$, and the closure of the modified $U_{0}$ is still $U$.

III. The Banach-Mazur theorem and Sierpiński's question. In [S], Sierpiński gave a proof that $C[0,1]$ is a universal separable metric space up to isometry. His proof was somewhat more elementary than the original proof of Banach and Mazur, but it still involved the use of a Hilbert cubefilling curve. Sierpiński was aware of the existence of $U$, which had been discovered by Urysohn earlier. Of course, Sierpiński's method of embedding a separable metric space isometrically in $C[0,1]$ can be applied to $U$; Sierpiński asked in $[\mathrm{S}]$ whether there was a "simpler or more natural" way to embed $U$ isometrically in $C[0,1]$ than the application of his general method. This is a vague question; what is simple or natural is often in the eye of the beholder. We can make some remarks. Sierpiński's use of a space-filling curve is not an accident; Lemma 2, Theorem 2 and the corollary which follows it, and the corollary to Lemma 5 should indicate to us that any embedding of $U$ in $C[0,1]$ has some relation to space-filling curves. The following modified version of Sierpiński's proof that $C[0,1]$ is a universal separable metric space makes the relation between the use of space-filling curves and the notion of "taking on half of all possible values" clearer. 
Definition. If $X$ is a metric space, and $x_{0}$ is a fixed point of $X$, let $\left(X, x_{0}\right)^{\prime}$ be the set of functions from $X$ to the reals such that $g\left(x_{0}\right)=0$ and $|g(x)-g(y)| \leq d(x, y)$ for each $x, y \in X$, i.e., the set of Lipschitz functions on $X$ with norm $\leq 1$ which send $x_{0}$ to 0 . We will usually call this $X^{\prime}$, where the identity of $x_{0}$ is understood. Note that if $X$ is a metric subspace of $C[0,1]$ containing $\mathbf{0},(X, \mathbf{0})^{\prime}$ is the set of possible combinations of values of $X$.

THEOREM 1. $C[0,1]$ is a universal separable metric space up to isometry.

Proof. Let $X$ be a separable metric space, and let $x_{0}$ be a fixed point of $X$. Put the pointwise convergence topology on $X^{\prime}$. Then $X^{\prime}$ is compact, because $|g(x)|=\left|g(x)-g\left(x_{0}\right)\right| \leq d\left(x, x_{0}\right)$, so $X^{\prime}$ is homeomorphic to a subset of the topological product of the intervals $\left[-d\left(x, x_{0}\right), d\left(x, x_{0}\right)\right]$ indexed by the points $x$ of $X$, which is compact, and $X^{\prime}$ is clearly a closed subset of this compact space, so is compact. $X^{\prime}$ is a metric space, because the values of a function in $X^{\prime}$ are completely determined by its values on a countable dense subset of $X$, so $X^{\prime}$ is homeomorphic to a subset of the product of the countable collection of intervals $\left[-d\left(x, x_{0}\right), d\left(x, x_{0}\right)\right]$ for $x$ in the countable dense subset of $X$, which is metrizable. It is straightforward to show that $X^{\prime}$ is a convex subset of $\mathbb{R}^{X}$ with the usual vector space structure, so it is a Peano continuum. Let $f$ be a map from $[0,1]$ onto $X^{\prime}$. Then the unique map $g$ such that $g(x)(t)=f(t)(x)$ is the desired isometry from $X$ into $C[0,1]$. Indeed, $d(g(x), g(y))$ is the supremum of expressions of the form $|g(x)(t)-g(y)(t)|=|f(t)(x)-f(t)(y)| \leq d(x, y)$, since $f(t)$ is an element of $X^{\prime}$. Since the function $h_{x}$ from $X$ to the reals which sends $y$ to $d(x, y)-d\left(x, x_{0}\right)$ is an element of $X^{\prime}$, there is $t \in[0,1]$ such that $f(t)=h_{x}$, and thus, for this value of $t,|g(x)(t)-g(y)(t)|=|f(t)(x)-f(t)(y)|=$ $\left|h_{x}(x)-h_{x}(y)\right|=\left|-d\left(x, x_{0}\right)-d(x, y)+d\left(x, x_{0}\right)\right|=d(x, y)$, proving that $d(g(x), g(y))=d(x, y)$, and $g$ is the desired isometry.

Note that it is actually not necessary for $f$ to fill $X^{\prime}$; it is sufficient for the image of $f$ to contain either $g$ or $-g$ for each $g \in X^{\prime}$, i.e. "half-fill" $X^{\prime}$, since $-h_{x}$ may be used as well as $h_{x}$ in the argument.

Sierpiński's proof can be understood in the terminology of our proof as constructing a path $f$ which visits each function $h_{x}$ instead of a path which visits every function in $X^{\prime}$. This is done by first taking a path filling the topological product of the intervals $\left[-d\left(x, x_{0}\right), d\left(x, x_{0}\right)\right]$ indexed by the points $x$ of $X$, then choosing for each $h_{x}$ a point in $[0,1]$ which maps to it and replacing the images of maximal open intervals of the complement of the closure of the set of selected points with "affine straight line segments". Since each $h_{x} \in X^{\prime}$ and $X^{\prime}$ is closed and convex, the image of the resulting path is in $X^{\prime}$. This procedure produces nicer embeddings of spaces simpler than $U$ than ours does; it turns out that when $X=U$, the set of $h_{x}$ 's is dense in $X^{\prime}$, which is not the case for most spaces $X$. 
The functions of $X^{\prime}$ can be viewed as "possible combinations of values" of functions in the image of $X$ in $C[0,1]$ under an isometry which sends $x_{0}$ to $\mathbf{0}$. In fact, every isometry $F$ from a metric space $X$ into $C[0,1]$ is associated with a path $G$ in $X^{\prime}$ by the relation $F(x)(t)=G(t)(x)$ for each $x \in X$ and $t \in[0,1]$. When is a path $G$ in $X^{\prime}$ associated with an isometry by this relation? For any path $G$ in $X^{\prime}$, there is a function $F$ from $X$ into $C[0,1]$ satisfying $F(x)(t)=G(t)(x)$. We have $d(F(x), F(y)) \leq d(x, y)$ by the argument used in the proof of Theorem 1 above, and $d(F(x), F(y))=d(x, y)$ exactly if there is a $t \in[0,1]$ such that $|F(x)(t)-F(y)(t)|=\mid G(t)(x)-$ $G(t)(y) \mid=d(x, y)$. Thus $F$ is an isometry exactly if the path $G$ meets the set $\left\{g \in X^{\prime}|| g(x)-g(y) \mid=d(x, y)\right\}$ for each $x, y \in X$, or, equivalently, if $G$ meets $\left\{g \in X^{\prime} \mid g(x)-g(y)=d(x, y)\right\}$ for each $x, y \in X$. This set can be thought of as a "face" of the "convex body" $X^{\prime}$ : this prompts the following definition.

Definition. Let facet $(x, y)$ be the set $\left\{g \in X^{\prime} \mid g(x)-g(y)=d(x, y)\right\}$, where $x$ and $y$ are points of a metric space $X$ whose identity is understood.

Thus we can summarize the discussion in the previous paragraph in the following economical form:

LEMMA. Any isometry $F$ from a metric space $X$ into $C[0,1]$ is associated with a path $G$ in $X^{\prime}$ such that $F(x)(t)=G(t)(x)$ for each $x \in X$ and $t \in[0,1]$. A path $G$ in $X^{\prime}$ is associated with an isometry in this manner if and only if it visits either facet $(x, y)$ or facet $(y, x)$ for each $x, y \in X$.

THEOREM 2. Every isometry from $U$ into $C[0,1]$ is of the type described in the proof of Theorem 1, as modified by the immediately following note.

Proof. If $X$ is taken to be a subspace of $C[0,1]$ containing $\mathbf{0}$, then $(X, \mathbf{0})^{\prime}$ is exactly the set of possible combinations of values of $X$. Thus, by the corollary to Lemma 5 of Part II, the path in $U^{\prime}$ associated with any isometry from $U$ into $C[0,1]$ sending $x_{0}$ to $\mathbf{0}$ visits $g$ or $-g$ for each $g \in U^{\prime}$, i.e., it half-fills $U^{\prime}$. But the isometric embeddings of $U$ in $C[0,1]$ constructed following the proof of Theorem 1 with the indicated modification are exactly those with this property.

Theorem 2 is the closest thing we know to a definite answer to Sierpiński's question cited above; we feel that our proof of Theorem 1 is a generalized version of Sierpiński's, and for our method of proof the answer is that there are no embeddings of $\left(U, x_{0}\right)$ in $(C[0,1], \mathbf{0})$ at all other than those derived from it. Sierpiński's method cannot generate every such isometry; in particular, it could not generate any isometry whose associated path mapped an open interval into the complement of the set of $h_{x}$ 's in a nonaffine manner. However, Sierpiński's technique can approximate any such isometry as closely as desired, if we allow the use of a curve which only "half-fills" a 
Hilbert cube. This theorem is definitely not true for any familiar space. For instance, the image of the path associated with the isometry from $\mathbb{R}$ into $C[0,1]$ sending each real to the corresponding constant function is a single point in $\mathbb{R}^{\prime}$; this set of functions takes on only one combination of values.

Here is a result which says something interesting about the geometry of $U^{\prime}$ :

TheOREM 3. For any open set $O$ in $U^{\prime}$, there are points $x, y \in U$ such that facet $(x, y)$ is contained in $O$.

Proof. A basis element of the pointwise convergence topology on $U^{\prime}$ is of the form $\left\{g \in U \mid g\left(x_{i}\right) \in\left(a_{i}, b_{i}\right)\right.$ for each $\left.i \leq n\right\}$, where $\left\{x_{1}, \ldots, x_{n}\right\}$ is a finite set of points in $U$ and $\left\{\left(a_{1}, b_{1}\right), \ldots,\left(a_{n}, b_{n}\right)\right\}$ is a finite collection of open intervals in the reals. Any open $O$ in $U^{\prime}$ contains a basis element, so we simply need to produce a facet inside this arbitrary basis element to prove the theorem. Let $h$ be an element of this basis element. Then $H=\left\{g \in U^{\prime} \mid g\left(x_{i}\right)=h\left(x_{i}\right)\right.$ for each $\left.i \leq n\right\}$ is a nonempty subset of the basis element. We claim that $H$ contains a facet. Let $R=(3 / 2) D$, where $D$ is the diameter of the set consisting of the $x_{i}$ 's and $x_{0}$. There must be a point $A$ which lies at distance $R-h\left(x_{i}\right)$ from each $x_{i}$, and distance $R$ from $x_{0}$, because this is a possible set of distances from the $x_{i}$ 's and $x_{0}$, since $h \in U^{\prime}$. There must be a point $B$ which lies at distance $R+h\left(x_{i}\right)$ from each $x_{i}$, at distance $R$ from $x_{0}$, and distance $2 R$ from $A$, for the same reason. Any function $f \in \operatorname{facet}(A, B)$ must satisfy $f(A)-f(B)=2 R$. But $f\left(x_{0}\right)$ must be 0 , and must differ from each of $f(A)$ and $f(B)$ by $\leq R$. The only way this can be true is if $f(A)=R$ and $f(B)=-R$. But then $f\left(x_{i}\right)$ must be $\leq f(B)+R+h\left(x_{i}\right)=h\left(x_{i}\right)$, and must also be $\geq f(A)-\left(R-h\left(x_{i}\right)\right)=R$, so $f\left(x_{i}\right)=h\left(x_{i}\right)$ for each $i \leq n$, and facet $(A, B)$ is a subset of $H$, which is a subset of our arbitrary basis element.

It would be easy to prove the corollary to Lemma 5 of Part II using Theorem 3 ; the associated path of any isometry from $U$ into $C[0,1]$ sending $x_{0}$ to $\mathbf{0}$ must visit each facet or its negative; thus, by Theorem 3 , it must visit each open set of $U^{\prime}$ or its negative, thus every point in $U^{\prime}$ or its negative. The points of $U^{\prime}$ are exactly the functions described in the corollary to Lemma 5 , and the associated path of an isometry visiting a certain point of $U^{\prime}$ is equivalent to the functions of that isometric copy of $U$ taking on the "combination of values" represented by that point of $U^{\prime}$.

Banach and Mazur's original proof that $C[0,1]$ is a universal separable metric space up to isometry actually established the following fact, which we state as a theorem without proof (see [B], [B-P]).

Theorem 4 (Banach and Mazur). $C[0,1]$ is a universal separable $B a$ nach space up to linear isometry. 
IV. The uniquely determined closed linear span of $U$. Now we come to some very surprising results about $U$. Nothing in the definition of $U$ would prepare us to believe that $U$ would have interesting properties in the context of linear spaces; it seems to be a strictly metric concept. However, it turns out that $U$ behaves in a very definite way in every Banach space in which it can be embedded. All results in this part are new.

TheOREM 1. When $U$ is embedded isometrically in any Banach space, with a point $x_{0}$ sent to the zero element of the Banach space, any finite subset of the isometric copy of $U$ which does not contain $x_{0}$ is linearly independent, and the linear closure $\mathbf{U}$ of the copy of $U$ is uniquely determined up to linear isometry.

Pr o of. We may without loss of generality assume that the Banach space in question is $C[0,1]$. For the linear closure of the separable space $U$ in any Banach space will itself be a separable Banach space, which by Theorem 4 of Part III can be considered to be embedded in $C[0,1]$.

We show that the closed linear span of $U$ is uniquely determined by demonstrating that the norm of each linear combination of elements of $U$ is exactly determined by the distances of the elements involved from $x_{0}$ and from one another. Thus the norm of any element of the closure of the set of linear combinations of elements of $U$ is exactly determined by the choice of $x_{0}$. By part (b) of the Theorem of Part I, $U$ has no privileged points, in the sense that there is an isometry from $U$ onto $U$ taking $x_{0}$ to any desired point $x$. Thus any isometric copy of $U$ in $C[0,1]$ which contains $\mathbf{0}$ can be carried to any other by an isometry which fixes $\mathbf{0 .}$

If $F=\left\{\mathbf{0}, f_{1}, \ldots, f_{n}\right\}$ is a finite subset of $C[0,1]$ and $a_{1}, \ldots, a_{n}$ are real numbers, then $\left\|\sum_{1 \leq i \leq n} a_{i} f_{i}\right\|=\sup _{t \in[0,1]}\left|\sum_{1 \leq i \leq n} a_{i} t^{*}\left(f_{i}\right)\right| \leq$ $\sup _{r \in F^{\prime}}\left|\sum_{1 \leq i \leq n} a_{i} r\left(f_{i}\right)\right|$, where $t^{*}$ is defined as the function which takes each element of $F$ to its value at $t$. By Lemma 2 of Part II, if $F$ is a subset of an isometric copy of $U$, for each $r \in F^{\prime}$ there is a $t \in[0,1]$ such that $r=t^{*}$ or $-r=t^{*}$. We can conclude that in this case we get $=$ above in place of $\leq$. If, in addition, we have $f_{i}=f_{j}$ iff $i=j$, we get an $r^{\prime} \in F^{\prime}$ defined by $r^{\prime}\left(f_{i}\right)=b a_{i} /\left|a_{i}\right|$, where $b>0$ is less than $d\left(f_{i}, f_{j}\right)$ for each pair of distinct indices $i$ and $j$. We then have $\left\|\sum_{1 \leq i \leq n} a_{i} f_{i}\right\|=$ $\sup _{r \in F^{\prime}}\left|\sum_{1 \leq i \leq n} a_{i} r\left(f_{i}\right)\right| \geq\left|\sum_{1 \leq i \leq n} a_{i} r^{\prime}\left(f_{i}\right)\right|=b \sum_{1 \leq i \leq n}\left|a_{i}\right|$, which is positive if any $a_{i}$ is nonzero; we see that any set of nonzero points of a copy of $U$ containing $\mathbf{0}$ is linearly independent. Moreover, since the expression $\left\|\sum_{1 \leq i \leq n} a_{i} f_{i}\right\|=\sup _{r \in F^{\prime}}\left|\sum_{1 \leq i \leq n} a_{i} r\left(f_{i}\right)\right|$ depends only on the $a_{i}$ 's and the distances between points in $\bar{F}$, it follows that any isometry between copies of $(U, \mathbf{0})$ in $C[0,1]$ extends linearly to an isometry between their linear spans and hence to a linear isometry between the closures thereof. 
Remark. It follows from our proof above that the norm of a finite linear combination of points of $U$, and thus the distance between two finite linear combinations of points of $U$, is as large as the distances among the points of $U$ involved and $x_{0}$ permit, subject to the axioms of a Banach space. Thus, another way of describing the norm of a linear combination $a$ of points of $U$ is as the minimum of expressions $\sum_{i}\left|c_{i}\right| d\left(a_{i}, a_{i}^{\prime}\right)$, where $a_{i}$ 's and $a_{i}^{\prime}$ 's are points of $U$ and $a=\sum_{i} c_{i}\left(a_{i}-a_{i}^{\prime}\right)$. A straightforward consequence of this is that it is possible to expand a distance $d(p, q)=\|p-q\|$ between finite linear combinations of points of $U$ as a minimal expression of the form $\sum_{i}\left|c_{i}\right| d\left(p_{i}, q_{i}\right)$ where $p=\sum_{i} p_{i}$ and $q=\sum_{i} q_{i}$, where $p_{i}, q_{i} \in U$ (this may require the introduction of cancelling terms into the expansion of the norm of $\|p-q\|$ described above).

Although no nontrivial linear combination of points of a copy of $U$ containing $\mathbf{0}$ is itself a point of $U$ (only linear combinations of the form $0 f$ or $1 f$, where $f$ is a point of $U$, can be points of $U$, by the linear independence of $U$ ), any linear combination of points in a copy of $U$ containing $\mathbf{0}$ is a point in some copy of $U$ containing $\mathbf{0}$; if $\left\{f_{1}, \ldots, f_{n}\right\}$ is a finite set of points in a copy of $U$ containing $\mathbf{0}$, then there is a function $g$ in the same copy of $U$ which inflates $\left\{f_{1}, \ldots, f_{n}\right\}$. Let $h$ be a linear combination of the $f_{i}$ 's and let $t, u$ be reals such that $|t| \leq 1,|u| \leq h$. Clearly, there exists an $x \in[0,1]$ and an $s^{\prime}= \pm 1$ such that $h(x)=s^{\prime} u$, and by the Remark preceding Lemma 3 of Part II there is a $y \in[0,1]$ such that $g(y)=s^{\prime \prime} s^{\prime} t$ and $f_{i}(y)=s^{\prime \prime} s^{\prime} f_{i}(x)$ for some $s^{\prime \prime}= \pm 1$. Then, also, $h(y)=s^{\prime \prime} s^{\prime} h(x)=s^{\prime \prime} s^{\prime} u$. By the Remark of Part II and Theorem 2 of Part II, $h$ belongs to some copy of $U$ containing $\mathbf{0 .}$

We now investigate the properties of the Banach space U. A natural question to raise is whether $\mathbf{U}$ is a universal separable Banach space up to linear isometry. We do not know the answer to this question; the rest of this paper covers our investigation of what Banach spaces can be embedded in $\mathbf{U}$. It would be easy to show that $\mathbf{U}$ was a universal separable Banach space if $\mathbf{U}$ had properties relative to Banach spaces similar to those that $U$ has relative to metric spaces; we show that $\mathbf{U}$ does not have such properties. We assume throughout that $U$ is a metric subspace of $C[0,1]$ containing $\mathbf{0}$, and thus that $\mathbf{U}$ is a closed linear subspace of $C[0,1]$.

THEOREM 2. $L_{1}$, the space of measurable functions on the nonnegative reals with the norm of a function equal to its Lebesgue integral over the nonnegative reals, can be embedded in a linear isometric fashion in $\mathbf{U}$.

P r o of. Let $I$ be an isometry from the nonnegative reals into $U$ such that $I(0)=\mathbf{0}$. Then the isometry which sends the characteristic function of the interval $[0, r]$ to $I(r)$ for each nonnegative real $r$ determines a linear isometry from $L_{1}$ into $\mathbf{U}$. The characteristic functions of intervals $[0, r]$ for nonzero $r$, which we denote by $X(r)$ below, are linearly independent in $L_{1}$ and their 
closed linear span is the whole space, so $I$ does in fact determine a linear map from $L_{1}$ into $\mathbf{U}$; it remains to show that this map is an isometry. It is sufficient to show that the norm of each finite linear combination of $X(r)$ 's is equal to the norm of the corresponding finite linear combination of $I(r)$ 's. Indeed, $\left\|\sum_{1 \leq i \leq n} a_{i} X\left(r_{i}\right)\right\|$, where we assume without loss of generality that $r_{i}<r_{j}$ iff $i<j$, can be expressed as $\sum_{1<i<n}\left|b_{i}\right|\left(r_{i}-r_{i-1}\right)$, where $r_{0}$ is taken to be 0 and $b_{i}=\sum_{i \leq j \leq n} a_{j}$. It is clear that $\left\|\sum_{1 \leq i \leq n} a_{i} I\left(r_{i}\right)\right\| \leq$ $\sum_{1 \leq i \leq n}\left|b_{i}\right|\left(r_{i}-r_{i-1}\right)$. To see that these quantities are equal, consider a $t \in[0,1]$ at which the functions $I\left(r_{i}\right)$ take on values $I\left(r_{i}\right)(t)=s f\left(r_{i}\right)$, where $s= \pm 1$ and $f$ is the continuous piecewise linear function with $f(0)=0$ and the slope of $f$ equal to $\left|b_{i}\right| / b_{i}$ in the interior of each interval $\left[r_{i-1}, r_{i}\right]$. This is clearly a possible combination of values for the $I\left(r_{i}\right)$ 's, so a $t$ exists which realizes it. The value of each $I\left(r_{i}\right)-I\left(r_{i-1}\right)$ at $t$ is $s\left(\left|b_{i}\right| / b_{i}\right)\left(r_{i}-r_{i-1}\right)$, so the value at $t$ of $\sum_{1 \leq i \leq n} b_{i}\left(I\left(r_{i}\right)-I\left(r_{i-1}\right)\right)$ is equal to $\sum_{1 \leq i \leq n} s\left|b_{i}\right|\left(r_{i}-r_{i-1}\right)$.

Corollary to Theorem 2. All two-dimensional Banach spaces can be embedded in $\mathbf{U}$ in a linear isometric fashion.

Proof. We show that all two-dimensional Banach spaces can be embedded in $L_{1}$ in a linear isometric fashion. (This result has previously been shown by Lindenstrauss in $[\mathrm{L}]$.)

With each Banach space $X$ with basis $\{u, v\}$ with $|u|=1$, we associate the function $f$ defined by $f(a)=d(a u, v)$. We investigate the properties of such functions $f$, and produce a characterization of such functions. From $f$, we can recover the Banach space $X:|a u|=|a|$ and $|a u+b v|=|b| f(-a / b)$ for $b \neq 0$. Applying the triangle inequality, we easily get $f(a+b) \leq f(a)+f(b)$ for $a, b \in \mathbb{R} ; f(c a+(1-c) b) \leq|c| f(a)+|1-c| f(b)$ for $a, b, c \in \mathbb{R}$. These conditions can be shown to characterize the class of such functions $f$ exactly. This analysis is analogous to our definition of "possible combinations of distances" in metric spaces, but we have only covered the analogue of the case of "possible distances" from one point $u$, which is completely trivial in the metric context! It follows that the graph of $f$ is concave up, so $f$ has a first derivative on the complement of a countable set which is nondecreasing, bounded above by 1 and below by -1 . Thus $f^{\prime}$ has a partial inverse $h$ which has domain $[-1,1]$ and is nondecreasing; fill in the graph of $h$ with horizontal lines corresponding to the vertical jumps in the graph of $f^{\prime}$. Define $g(t)$ as $h((t+1) / 2)$ and let $X(s)$ denote the characteristic function of $[0, s]$.

We will compute $d(a X(1), g)$ using the integral metric of $L_{1}[0,1]$ and show that it exists for each $a$; the case $a=0$ verifies that $g \in L_{1}$.

Observe that $\int_{[0,1]}|g-a|$, the integral which, if it exists, will be the desired distance, is $(1 / 2)\left(\int_{[a, \infty]}\left(1-f^{\prime}\right)-\int_{[-\infty, a]}\left(f^{\prime}+1\right)\right)$, by an obvious 
operation on the graph. Now,

$$
\begin{aligned}
& \frac{1}{2}\left(\int_{[a, b]} 1-f^{\prime}\right)=\frac{1}{2}(b-a-(f(b)-f(a))), \\
& \frac{1}{2}\left(\int_{[-c, a]} f^{\prime}+1\right)=\frac{1}{2}(a+c+f(a)-f(-c)) .
\end{aligned}
$$

If we add the approximations to the improper integrals, we obtain $(1 / 2)(b-$ $f(b)+c-f(-c)+2 f(a))$.

The limit as $b$ approaches infinity of $b-f(b)$ and the limit as $c$ approaches infinity of $c-f(-c)$ both exist and are bounded in absolute value by $|v|$; $b-f(b)$, for instance, is equal to $d(\mathbf{0}, b u)-d(v, b u) \in[-|v|,|v|]$ and must increase. We term these limits $r_{1}$ and $r_{2}$, respectively. Moreover, $b-f(b)+$ $c-f(-c)=b+c-(f(b)+f(-c))=b+c-(d(v, b u)+d(v,-c u)) \leq 0$. If we set $r=-\left(r_{1}+r_{2}\right) / 2$, we see that $d(a X(1), g)=f(a)-r=d(a u, v)-r$, and $r \geq 0$. The map $F$ which sends $u$ to $X(1) \in L_{1}[0,2]$ and $v$ to $g+r(X(2)-X(1)) \in$ $L_{1}[0,2]$ satisfies $d(F(a u), F(v))=\|a u-v\|$ and hence extends linearly to a linear isometry from $X$ into $L_{1}[0,2]$.

Definition. We say that a point $b$ in a metric space is between points $a$ and $c$ iff $d(a, b)+d(b, c)=d(a, c)$. We define $B(a, c)$ as the set of points $b$ which are between $a$ and $c$. The set of points between $a$ and $b$ in a metric space which lie in a subspace $X$ will be denoted by $B_{X}(a, b)$.

A triple midpoint between points $a, b$, and $c$ is a point $d$ which is in the intersection of $B(a, b), B(a, c)$ and $B(b, c)$. It is straightforward to establish that a triple midpoint between $a, b$, and $c$ is at distance $(d(a, b)+d(a, c)-$ $d(b, c)) / 2$ from $a$ and symmetrically determined distances from $b$ and $c$. A metric space has the triple midpoint property if any three points in the space have at least one triple midpoint.

$U$ obviously has the triple midpoint property. Any space $C[X]$ has the triple midpoint property, because it is easy to show that for any combination of distances $r$ from a set $S$, there is a point $t$ such that $d(s, t) \leq r(s)$ for each $s \in S$, and the combination of distances from $a, b$, and $c$ at which a triple midpoint would be found is minimal. Any $l_{1}$ space, that is, a function space with a sum or integral metric, has unique triple midpoints - for each triple of points $a, b, c$ there is exactly one triple midpoint. We sketch a proof for a space of functions with the integral metric: if $f, g$, and $h$ are functions in the space, it is straightforward to show that the unique function whose value at each $t$ is in the closed intervals between $f(t)$ and $g(t)$, between $f(t)$ and $h(t)$, and between $g(t)$ and $h(t)$ is the unique triple midpoint between $f, g$, and $h$. We prove two lemmas, then we prove that $\mathbf{U}$ does not have the triple midpoint property. 
Lemma 1. Let $A$ and $B$ be subsets of $U$ with finite diameter such that for some positive $e, d(a, b)>e$ for each $a \in A$ and $b \in B$. Then the intersection of the closed linear span of $A$ and the closed linear span of $B$ is $\{\mathbf{0}\}$. (Note that the conditions of the lemma hold in the case where $A$ and $B$ are disjoint compact subsets of $U$.)

Proof. Let $a$ and $b$ be points in the closed linear spans of $A, B$, respectively. Let $e$ be a positive real number. Let $a^{\prime}, b^{\prime}$ be finite rational linear combinations of points of $A, B$, respectively, within distance $e$ of $a, b$ respectively. Expand $d\left(a^{\prime}, b^{\prime}\right)$ as indicated in the Remark above, as a minimal expression of the form $\sum_{i}\left|c_{i}\right| d\left(a_{i}^{\prime}, b_{i}^{\prime}\right)$, where $\sum_{i} c_{i} a_{i}^{\prime}=a^{\prime}$ and $\sum_{i} c_{i} b_{i}^{\prime}=b^{\prime}$. Using the fact that all coefficients in the expansions of $a^{\prime}$ and $b^{\prime}$ are rational, we may assume that all $c_{i}$ 's have the same absolute value $c$ (the gcd of all coefficients occurring in the original expansions of $a^{\prime}$ and $b^{\prime}$ ). It is also possible to assume without loss of generality that wherever a pair of terms $c_{i} a_{i}^{\prime}$ and $c_{j} a_{j}^{\prime}$ satisfy $c_{i}=-c_{j}$ and $a_{i}^{\prime}=a_{j}^{\prime} \neq \mathbf{0}$ then either $b_{i}^{\prime}=a_{i}^{\prime}=a_{j}^{\prime}$ or $b_{j}^{\prime}=a_{j}^{\prime}=a_{i}^{\prime}$; otherwise, it would be possible to replace both $a_{i}^{\prime}$ and $a_{j}^{\prime}$ with either $b_{i}^{\prime}$ or $b_{j}^{\prime}$ without increasing the value of the expansion of $d\left(a^{\prime}, b^{\prime}\right)$ (by an application of the triangle inequality) and thus without changing it, since the value of the expansion is minimal for expansions of the same form (the replacement does not affect the value of $a^{\prime}$ because the terms involved cancel one another). Such a process of substitution can be iterated and must terminate. Note that it is also possible to assume that all $a_{i}^{\prime}$ 's not equal to $\mathbf{0}$ occur nontrivially in the expansion of either $a^{\prime}$ or $b^{\prime}$ (cancelling pairs of terms involving points from the expansion of $b^{\prime}$ may be involved) - all other points have to occur in cancelling pairs of terms which can be eliminated by possibly iterated application of the technique indicated above. All these remarks apply by symmetry to the expansion of $b^{\prime}$ as well.

It follows that each point $a_{i}^{\prime}, b_{i}^{\prime}$ is in $A$, in $B$, or equal to $\mathbf{0}$. Each term $c d\left(a_{i}^{\prime}, b_{i}^{\prime}\right)$ of the expansion of $d\left(a^{\prime}, b^{\prime}\right)$ such that one of the points is in $A$ and the other is in $B$ makes a contribution of at least $c d(A, B)$ to $d\left(a^{\prime}, b^{\prime}\right)$. The sum of the absolute values of the coefficients of such $a_{i}^{\prime}$ 's and $b_{i}^{\prime}$ 's will be as small as desired if $d\left(a^{\prime}, b^{\prime}\right)$ is taken to be sufficiently small (as it will be if $d(a, b), e$ are taken to be sufficiently small). Since the sets $A$ and $B$ are bounded, it follows that the points $a^{\prime \prime}, b^{\prime \prime}$ obtained by eliminating all such terms from the expansions of $a^{\prime}, b^{\prime}$ respectively are as close as desired to $a^{\prime}, b^{\prime}$, respectively, under the same conditions. Eliminating the corresponding terms from the expansion of $d\left(a^{\prime}, b^{\prime}\right)$ gives an expansion of $d\left(a^{\prime \prime}, b^{\prime \prime}\right)$ (a "better" candidate for the expansion of this distance could be used to give a "better" candidate for the expansion of $d\left(a^{\prime}, b^{\prime}\right)$ ); now observe that since points of $A$ and $B$ never correspond to one another in the expansions of $a^{\prime \prime}, b^{\prime \prime}$, the expansion of $d\left(a^{\prime \prime}, b^{\prime \prime}\right)$ can be divided into two expansions, one 
involving points of $A$ and $\mathbf{0}$ and one involving points of $B$ and $\mathbf{0}$, which are easily seen to be expansions of $d\left(a^{\prime \prime}, \mathbf{0}\right)$ and $d\left(b^{\prime \prime}, \mathbf{0}\right)$, since $a^{\prime \prime}$ involves points of $B$ only trivially and $b^{\prime \prime}$ involves points of $A$ only trivially. If these were not actually expansions of the distances indicated, a "better" expansion could be found for $d\left(a^{\prime \prime}, b^{\prime \prime}\right)$. Thus $d\left(a^{\prime \prime}, b^{\prime \prime}\right)=d\left(a^{\prime \prime}, \mathbf{0}\right)+d\left(b^{\prime \prime}, \mathbf{0}\right)$, and it follows that forcing $a$ and $b$ closer together and $e$ to 0 , which clearly forces $d\left(a^{\prime \prime}, b^{\prime \prime}\right)$ to 0 , thus forces $a^{\prime \prime}$ and $b^{\prime \prime}$ to $\mathbf{0}$-but this also forces $a$ and $b$ themselves to $\mathbf{0 .}$

LEMMA 2. Let $p$ and $q$ be points of $U$. Then any point of $\mathbf{U}$ between $p-q$ and $\mathbf{0}$ is in the closed linear span of $B_{U}(p, q)$.

Proof. Note that $h$ is between $\mathbf{0}$ and $p-q$ iff $h+q$ is between $p$ and $q$, and $h+q$ is in the closed linear span of $B_{U}(p, q)$ iff $h$ is. It follows that it is equivalent to prove that points between $p$ and $q$ are in the closed linear span of the set of points of $U$ between $p$ and $q$, and this is what we will prove.

Let $h$ be a point of $\mathbf{U}$ between $p$ and $q$. For any $e>0$, we can find $h^{\prime}$ within distance $e / 2$ of $h$ which is a finite linear combination of points of $U$ with rational coefficients. Expand the distances from $p$ to $h^{\prime}$ and from $h^{\prime}$ to $q$ in the form described in the Remark and used in the proof of Lemma 1. As in the proof of Lemma 1, we can assume without loss of generality that the absolute values of all coefficients are the same (call this value $c$ ). We may suppose without loss of generality that the expansions of $h^{\prime}$ involved in the two expansions of distances are the same.

We now construct sequences of terms from the expansions of $d\left(p, h^{\prime}\right)$ and $d\left(h^{\prime}, q\right)$, which we will refer to as paths. The paths will be disjoint and will contain no term more than once. A path will have as its first term a term $\left|c_{i}\right| d\left(p_{i}, h_{i}^{\prime}\right)$ of the expansion of $d\left(p, h^{\prime}\right)$ such that $c_{i}=c$ and $p_{i}=p$; a $(4 n+1)$ st term of a path will be a term $\left|c_{i}\right| d\left(p_{i}, h_{i}^{\prime}\right)$ of the expansion of $d\left(p, h^{\prime}\right)$, the $(4 n+2)$ nd term will be the corresponding term $\left|c_{i}\right| d\left(h_{i}^{\prime}, q_{i}\right)$ of the expansion of $d\left(h^{\prime}, q\right)$, the $(4 n+3)$ rd term will be a term $\left|c_{j}\right| d\left(h_{j}^{\prime}, q_{j}\right)$ such that $c_{j}=-c_{i}$ and $q_{i}=q_{j}$, and the $(4 n+4)$ th term will be $\left|c_{j}\right| d\left(p_{j}, h_{j}^{\prime}\right)$. The $(4 n+5)$ th term, if there is one, must be a term $\left|c_{k}\right| d\left(p_{k}, h_{k}^{\prime}\right)$ such that $c_{k}=-c_{j}=c_{i}$ and $p_{j}=p_{k}$. In the course of constructing paths, we reserve the right to introduce additional zero terms in all expansions if this enables us to lengthen paths nontrivially; this has the effect that each path will actually begin with a term $c d\left(p, h_{i}^{\prime}\right)$ and end with a term $c d\left(h_{j}^{\prime}, q\right)$. The reason for this is that the only terms in the expansions of $p, q$ respectively which do not have cancelling terms are of the form $c p, c q$ respectively. We extend paths whenever possible subject to the condition that each term is only used once in any path, and whenever a path terminates, we start constructing a new path if a suitable first term is still available. When the process is complete, $1 / c$ paths will have been constructed. There may 
remain terms which are not on any path. The sum of the terms in the expansion of $p$ not involved in any path will be zero, and likewise for $q$; this will not necessarily be true of $h^{\prime}$, but we will show that the norm of the sum of such terms of $h^{\prime}$ is less than $e$.

We define the length of a path as the sum of the terms on the path. By iterated applications of the triangle inequality, the length of each path must be at least $c d(p, q)$, so the sum of the lengths of all paths must be at least $d(p, q)$. Now if we let $H$ represent the sum of all terms of the expansion of $h^{\prime}$ which are not on any path, we observe that the sum of all terms of the expansion of $d\left(p, h^{\prime}\right)$ which involve terms of $h^{\prime}$ and $p$ not on any path is an expansion of $d(H, \mathbf{0})$, and similarly for $d\left(h^{\prime}, q\right)$. Thus the norm of $H$ is bounded above by the excess of $d\left(h^{\prime}, p\right)+d\left(h^{\prime}, q\right)$ over $d(p, q)$, which is bounded in turn by $e$.

Now fix a positive real number $d$ and observe that each term of the expansion of $h^{\prime}$ which occurs on a path and is at a distance greater than $d$ from $B_{U}(p, q)$ must make a contribution of at least $c d$ to the excess of the length of the path it is on over $c d(p, q)$. This implies that for $e$ small enough and $N$ the number of paths containing points at a distance greater than $d$ from $B_{U}(p, q)$, the quantity $N c$ will be as small as desired. Now observe that the terms of $p$ involved in such paths will add up to $N c p$ and the terms of $q$ involved in such expansions will add up to $N c q$, while, if we let $H^{\prime}$ represent the total of points of the expansion of $h^{\prime}$ involved in such paths, the total length of these paths will be an expansion of $d\left(H^{\prime}, N c p\right)+d\left(H^{\prime}, N c q\right)$, a quantity which approaches $2 d\left(H^{\prime}, \mathbf{0}\right)$ as $e$ and $N c$ approach zero. The total length of paths which do not involve points at a distance greater than $d$ from $B_{U}(p, q)$ will be at least $(1-N c) d(p, q)$. As $e$ approaches zero, $N c$ approaches zero, and the total length of paths not containing points at a distance greater than $d$ from $B_{U}(p, q)$ will approach a quantity greater than or equal to $d(p, q)$, so the sum of all other terms of the expansion, which approaches $2 d(H, \mathbf{0})+2 d\left(H^{\prime}, \mathbf{0}\right)$, will approach zero. This implies that the distance between $h^{\prime}$ and the sum of the terms of its expansion which are within $d$ of $B_{U}(p, q)$ will become as small as desired if $e$ is taken sufficiently small. We can obtain a point within $d$ of this point which is a point of the linear span of $B_{U}(p, q)$ by replacing each point in the expansion with a point within distance $d$ which is actually in $B_{U}(p, q)$. Thus, if we take first $d$ and then $e$ small enough, we can obtain a point as close to the original $h$ as desired which is a finite linear combination of points of $B_{U}(p, q)$.

THEOREM 3. U does not have the triple midpoint property.

Proof. Consider points $a, b, c, d$ of $U$, with $d(a, b)=d(c, d)=1$, $d(b, c)=d(d, a)=1 / 2$, and $d(a, c)=d(b, d)=3 / 2$. (Such points can be found on a circle of circumference 3 with the distance between two points 
defined as the shortest arc distance.) The distances of all these points from $\mathbf{0}$ can be taken to be $3 / 4$. Define $A$ as $a-b$ and $B$ as $d-c$. Then $d(A, \mathbf{0})=$ $d(B, \mathbf{0})=1$, obviously. Also, $d(A, B) \leq d(a, d)+d(b, c)+1$, and the values of $A$ and $B$ actually differ by 1 when $a, c$ take on the value $1 / 4$ and $b, d$ take on the value $-1 / 4$, so $d(A, B)=1$ as well. If $A, B$, and $\mathbf{0}$ have a triple midpoint $C$, we must have $d(A, C)=d(B, C)=d(\mathbf{0}, C)=1 / 2$. Finally, observe that the intersection of $B_{U}(a, b)$ and $B_{U}(c, d)$ is empty; if a point lies in $B_{U}(a, b)$, it must be at distance $\leq 1 / 2$ from either $a$ or $b$, and thus at distance $\geq 1$ from either $c$ or $d$. The only apparently possible case is that in which these inequalities are actually equations, so the point in the intersection of $B_{U}(a, b)$ and $B_{U}(c, d)$ is a midpoint between $a$ and $b$ and is actually $c$ or $d$ itself; but neither $c$ nor $d$ is a midpoint between $a$ and $b$. Now observe that the closed linear spans of $B_{U}(a, b)$ and $B_{U}(c, d)$ have intersection $\{\boldsymbol{0}\}$ by Lemma 1 , and that the triple midpoint $C$ of $A, B$, and 0 must be in the intersection of these closed linear spans by Lemma 2, which is impossible, because the distance from $C$ to $\mathbf{0}$ is supposed to be $1 / 2$.

The result of Theorem 3 shows that $\mathbf{U}$ contains a collection of three points and a combination of distances from those points which is permitted by the axioms of a Banach space (it is realized in $C[0,1]$ for those three points) which is not realized by a point in $\mathbf{U}$. This rules out a proof that $\mathbf{U}$ is a universal separable Banach space which would be analogous to the proof that $U$ is a universal separable metric space.

A natural line of investigation to pursue at this point is to attempt to determine what spaces $\mathbb{R}^{n}$ with the supremum metric can be linearly isometrically embedded in $\mathbf{U}$. If all such spaces could be embedded in $\mathbf{U}$, we could conclude that all finite-dimensional Banach spaces with polyhedral unit balls could be embedded in $\mathbf{U}$ (this follows from results about the geometry of convex polytopes: see $[G])$. It proves possible to embed $\mathbb{R}^{2}, \mathbb{R}^{3}$, and $\mathbb{R}^{4}$ with the supremum metric in a linear isometric fashion in $\mathbf{U}$, but it is not clear whether all such spaces can be embedded in $\mathbf{U} . \mathbb{R}^{4}$ with the supremum metric is embedded as follows: choose points $a, a^{\prime}, b, b^{\prime}, c, c^{\prime}, d$, $d^{\prime}$ in $U$ such that the distance between each of the first four points and each of the last four points is $1 / 4$ and each other nontrivial distance is $1 / 2$. The basis elements for a linear isometric copy of $\mathbb{R}^{4}$ with the supremum metric are $a-a^{\prime}+b-b^{\prime}, a-a^{\prime}-b+b^{\prime}, c-c^{\prime}+d-d^{\prime}$, and $c-c^{\prime}-d+d^{\prime}$.

Acknowledgements. I am grateful to my graduate advisors, Louis F. McAuley and the late Prabir Roy, for encouraging me to work on this topic; I am also indebted to Professor Henryk Toruńczyk for his excellent advice on revising the paper, without which no one would have been able to read it! 


\section{References}

[B] S. Banach, Théorie des Opérations Linéaires, Hafner, New York 1932, p. 185.

[B-P] C. Bessaga and A. Pełczyński, Selected Topics in Infinite-dimensional Topology, Monograf. Mat. 58, Polish Scientific Publishers, Warszawa 1975, pp. 48-51.

[G] B. Grünbaum, Convex Polytopes, Wiley, London 1967, pp. 72-73.

[H] G. E. Huhunaishvili, A property of the universal metric space of Urysohn, Dokl. Akad. Nauk SSSR 101 (1955), 607-610 (in Russian).

[J] C. Joiner, On Urysohn's universal separable metric space, Fund. Math. 73 (1971), $51-58$.

[L] J. Lindenstrauss, On the extension of operators with a finite-dimensional range, Illinois J. Math. 8 (1964), 488-499.

[S] W. Sierpiński, Sur un espace métrique séparable universel, Fund. Math. 33 (1945), 115-122. Also see his General Topology, Univ. of Toronto Press, Toronto 1952, pp. 159-162.

[U] P. Urysohn, Sur un espace métrique universel, Bull. Sci. Math. 51 (1927), 43-64, 74-90.

[Z] M. Ziegler, Ein problem von Urysohn, preprint, 1978.

DEPARTMENT OF MATHEMATICS

BOISE STATE UNIVERSITY

1910 UNIVERSITY DRIVE

BOISE, IDAHO 83725

U.S.A.

Received 10 June 1990;

in revised form 3 January 1991 and 16 August 1991 